\title{
A Hybrid Coarse/Fine Layered Multicast Scheme Based on Hierarchical Bandwidth Inference Congestion Control
}

\author{
Jian-Liang Lin, Wen-Liang Hwang, Soo-Chang Pei, Fellow, IEEE, and Jenq-Neng Hwang, Fellow, IEEE
}

\begin{abstract}
Traditional approaches to receiver-driven layered multicast apply coarse-grain layered congestion control, which enables each receiver to adjust its receiving rate to match the available bandwidth. However, this restricts the scalability of fine-grain scalable video coding. In this paper, we present an approach that facilitates bandwidth inference congestion control in a hybrid coarse/fine layered multicast scheme for multimedia delivery. One-way delay trend detection has proven effective in explicitly or implicitly estimating end-to-end available bandwidth. Using a hierarchical layered probing scheme and a delay trend detection method, we have developed a congestion control protocol for fine-grain layered multicast. Combining this protocol with scalable video coding, we present a framework that achieves efficient scalable video streaming over heterogeneous networks. The performance of our hybrid coarse/fine layered multicast scheme is evaluated by network simulations and the video coding efficiency.
\end{abstract}

Index Terms-Available bandwidth estimation, congestion control, hybrid coarse/fine layered multicast.

\section{INTRODUCTION}

$\mathbf{M}$ ULTIMEDIA communication over (wired or wireless) IP networks has become increasingly important due to the explosive growth of the Internet. Scalability at the bit-stream level is an important feature of multimedia delivery over networks. The objective of a scalable video coding scheme is to encode a video signal once, and enable different receivers to decode the video by receiving only part of the bit-stream, depending on the available bandwidth and the target resolution. MPEG-4 fine-grain scalability (FGS) provides a scalable video coding scheme based on hybrid motion compensation and bit-plane DCT coding [15]. In [5], a fine-grain scalable video coding scheme based on hybrid motion compensation and bit-plane coding of matching pursuit atoms was proposed to achieve better coding efficiency at low bit rates. However, to avoid the drifting problem, hybrid motion-compensation-based scalable video coding algorithms sacrifice coding efficiency by only using the base layer for motion compensation. Some

Manuscript received September 01, 2005; revised September 27, 2006. First published September 16, 2008; current version published November 26, 2008. This paper was recommended by Associate Editor J.-R. Ohm.

J.-L. Lin was with the Institute of Communications Engineering, National Sun Yat-Sen University, Kaohsiung 804, Taiwan. He is now with MediaTek Inc., Hsinchu 300, Taiwan. (e-mail: jlin@ faculty.nsysu.edu.tw).

W.-L. Hwang is with the Institute of Information Science, Academia Sinica, Taipei 115, Taiwan, and also with Digital Technology Department, Kainan University, Taoyung 33857, Taiwan. (e-mail: whwang@iis.sinica.edu.tw).

S.-C. Pei is with the Department of Electrical Engineering, National Taiwan University, 10617 Taiwan. (e-mail: pei@cc.ee.ntu.edu.tw).

J.-N. Hwang is with the Department of Electrical Engineering, University of Washington, Seattle, WA 98105 USA (e-mail: hwang@ee.washington.edu).

Color versions of one or more of the figures in this paper are available online at http://ieeexplore.ieee.org.

Digital Object Identifier 10.1109/TCSVT.2008.2004929 efficient scalable video coding schemes based on motion-compensated temporal filtering (MCTF) have been proposed to improve coding efficiency, achieve scalability, and solve the drifting problem encountered in closed-loop hybrid coding systems [1], [2], [6].

Layered multicasting with a scalable video coding scheme enables efficient dissemination of real-time multimedia streaming over heterogeneous networks [8]. In a layered multicast system, the server divides a scalable bit-stream into different layers, each of which is disseminated by a separate IP multicast group. In order to adapt to the rapidly changing available network throughput, receivers must be able to estimate the available bandwidth in real-time and dynamically join the appropriate cumulative layers. Several receiver-driven layered multicast protocols have been proposed to improve intra-session fairness so that each receiver gets the quality of video commensurate with network bandwidth availability and the end-system processing load [4], [9], [10], [14], [16]. Based on a one-way delay trend detection technique, a bandwidth inference congestion (BIC) control protocol for multimedia layered multicast is proposed in [12]. Each receiver uses one-way delay trend detection to estimate the end-to-end available bandwidth.

In this paper, based on the BIC control protocol, we propose a hierarchical bandwidth inference congestion control scheme that can achieve hybrid coarse/fine layered multicast. The remainder of the paper is organized as follows. In Section II, we briefly describe scalable video coding schemes based on MCTF and discuss the one-way delay trend detection model and BIC control for layered multicast. In Section III, we propose a hierarchical probing scheme for a hybrid coarse/fine layered multicast system. Section IV presents the experiment results of the proposed hierarchical layered multicast scheme. Then, in Section V, we present our conclusions.

\section{PRevious Work}

\section{A. Scalable Video Coding With MCTF}

In recent years, several efficient scalable video coding schemes based on motion-compensated temporal filtering (MCTF) have been proposed [1]-[3], [6], [7]. Three-dimensional (3-D) wavelet scalable video coding is one of the most popular techniques for achieving scalability in video coding, because it has embedded coding characteristics and the ability to achieve continuous rate scalability. Another major scalable video coding scheme based on an extension of H.264/AVC is proposed in [2] and [3].

Temporal scalability is provided by MCTF and hierarchical B-frames, whereas spatial scalability is provided by upsampling. For signal-to-noise ratio (SNR) scalability, two types of scalability, coarse-grain and fine-grain, are provided. The former can be achieved easily by using different quantization 


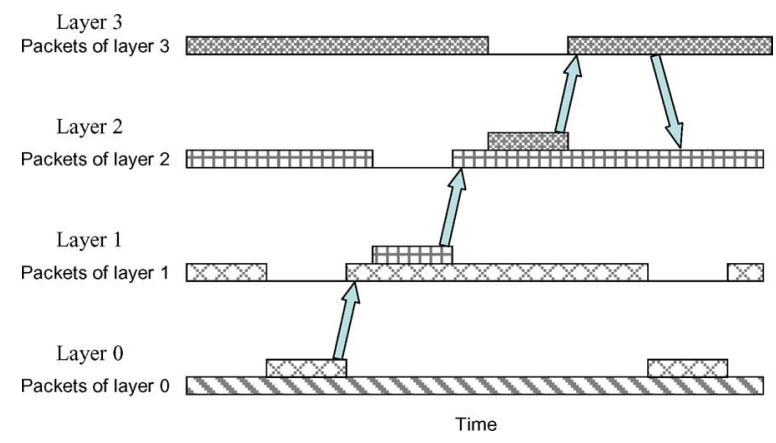

Fig. 1. Example of the BIC control scheme for layered multicast.

steps to code the DCT coefficients, while the latter can be achieved by encoding successive refinements of subbitplane arithmetic coding. Combined with the concepts of temporal, spatial, and SNR scalability, the scalable video coding scheme based on the extension of H.264/AVC and MCTF can efficiently generate a bit-stream with a high degree of temporal, spatial, and SNR scalability.

\section{B. Delay Trend Detection and BIC Control}

In recent years, one-way delay trend detection techniques have been proposed to detect potential end-to-end network congestion and estimate end-to-end available bandwidth. In [12] and [13], Liu et al. use a delay trend detection technique to estimate end-to-end available bandwidth and propose a bandwidth inference congestion (BIC) control protocol for multimedia applications. By sending a probe at a fixed-rate and analyzing the one-way delay trend, the receiver can determine whether the available bandwidth is higher than the probing rate. This scheme checks the available bandwidth by sending a probe at the target bit rate and detecting the one-way delay trend. The advantage of one-way delay trend detection is that it does not require synchronized clocks. If the sender and receiver do not have synchronized clocks, as long as the clock offset is constant, the one-way delay trend of the packets will not be affected. There are no special requirements for the probe packets, which can be audio or video data of variable-size in practical multimedia applications.

In the proposed BIC control scheme for multilayered multicast, a sender periodically probes each layer by sending packets from the upper layer to the current layer. Receivers can then test the probing bit rate and decide whether or not to join an additional layer according to the delay trend detected. Joining an additional layer depends on the one-way delay trend detected during the probing period, whereas, leaving a layer is based on packet loss. Fig. 1 illustrates an example of a four-layer multicast, where the server probes from layer 0 to layer 2 periodically. The arrows show a receiver from layer 0 joining layers 1,2 , and 3 after three probes, and then leaving layer 3 because of packet loss.

\section{A Hierarchical Probing Scheme For Hybrid COARSE/FINE LAYERED MULTICAST}

In the BIC control scheme for layered multicast described in the previous section, the scale of the layers should not be too

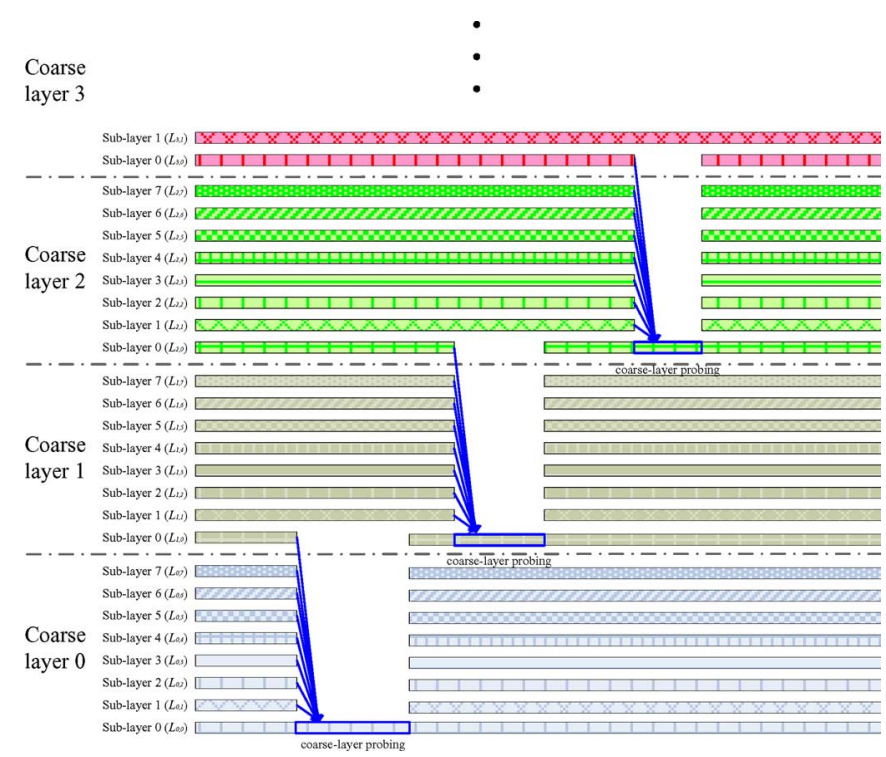

Fig. 2. Periodical coarse-layer probing structure of the hybrid coarse/fine layered scheme.

small; otherwise, it will take a long time to reach the optimal layer if the available bandwidth has large increments. The objective of scalable video coding is to support fine-grain scalability; however, coarse-grain layered multicast protocol restricts scalability. The difficulties associated with layered scalability and convergence time motivate us to develop a hierarchical probing scheme to achieve a hybrid coarse/fine layered multicast system.

Suppose the layered multicast system consists of $N$ coarse layers, $\left\{L_{n}, n=0,1, \ldots, N-1\right\}$, each of which can be further divided into $M$ fine-grain layers called sublayers. $M$ can be different for each coarse layer. An example of the hybrid coarse/fine layered multicast structure with eight sublayers is shown in Fig. 2. We denote $L_{n, m}$ as the $m$ th sublayer in the $n$th coarse layer and $R_{n, m}$ as the cumulative rate up to layer $L_{n, m}$. A receiver at layer $L_{n, m}$ receives packets from coarse layers 0 to $n-1$ and packets from sublayers 0 to $m$ in coarse layer $n$. In coarse-layered probing, the server periodically probes the coarse layers $0,1, \cdots, N-2$, as shown in Fig. 2 . When a coarse layer $n$ is being probed, packets belonging to $\left\{L_{n, j} \mid j=1,2, \ldots, M-1\right\}$ and $L_{n+1,0}$ (the first sublayer of the upper coarse layer) are sent through sublayer $L_{n, 0}$ (the first sublayer of the current coarse layer). Thus, all receivers subscribing to coarse layer $n$ are forced to receive the probe packets at target bit rate $R_{n+1,0}$, which is the bit rate of the first sublayer of the coarse layer $n+1$. Each receiver then uses delay trend detection to decide whether the end-to-end available bandwidth is higher than the probing bit rate $R_{n+1,0}$. If a receiver at $L_{n, m}$ detects that its available bandwidth is higher than the probing bit rate, it will subscribe to layer $L_{n+1,0}$, i.e., it will join multiple additional layers up to $L_{n+1,0}$; otherwise, it will stay at its original layer, $L_{n, m}$.

In this probing scheme, the coarse layers are probed in sequence. After a coarse layer $n$ has been probed and stabilized, the server waits for an interval before starting hierarchical sublayer probing in that layer. The purpose of this probing is to enable receivers to find an optimal fine-grain layer close to their 


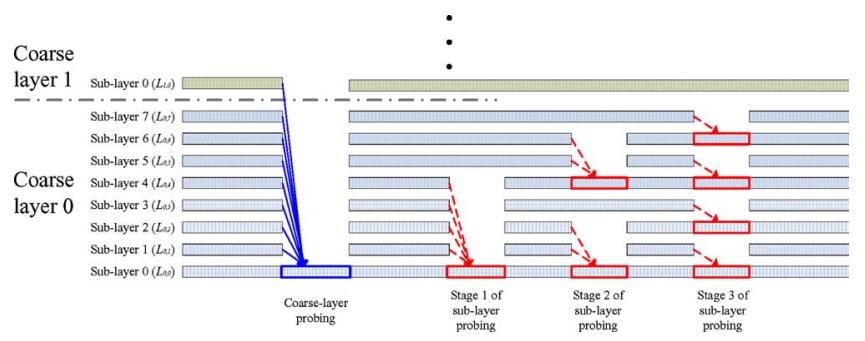

Fig. 3. Structure of the hierarchical layered probing scheme for hybrid coarse/fine layered multicast, where the number of sublayers $M=2^{3}$. The solid blue lines represent the probes of the coarse layers, while the dashed red lines represent the hierarchical sublayer probes.

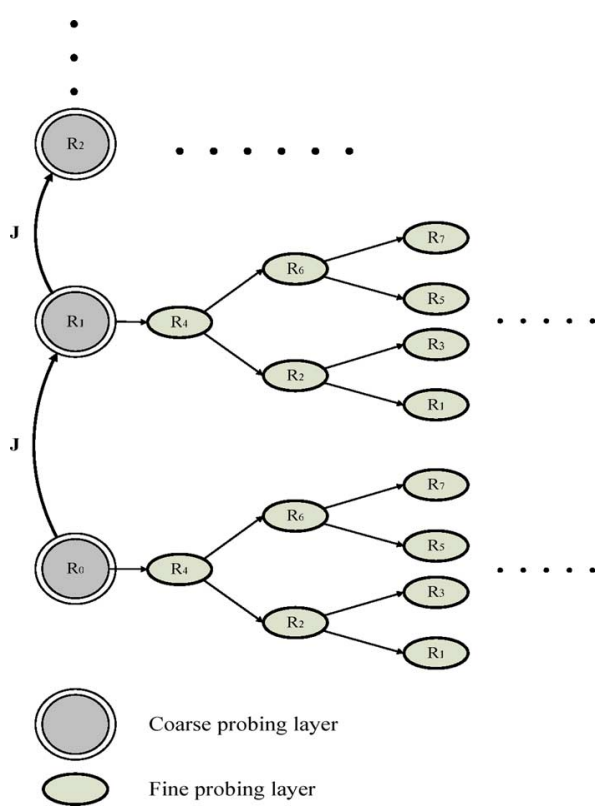

Fig. 4. Hybrid coarse/fine layered probing scheme.

actual available bandwidth. In the following, we give a detailed description of the implementation of the scheme.

The hierarchical probing structure of a coarse layer $n$ comprised of $M$ sublayers has $Q=\left\lfloor\log _{2} M\right\rfloor$ stages in the hierarchical sublayer probing circle, as shown in Fig. 3. In the first step of sublayer probing at coarse layer $n$, the server sends the probe packets at the bit rate of the medium sublayer, $R_{n, 2 Q-1}$ (the bit rate of sublayer $L_{n, 2^{Q-1}}$ ), by sending packets of $\left\{L_{n, j} \mid j=\right.$ $\left.1,2, \ldots, 2^{Q-1}\right\}$ through sublayer $L_{n, 0}$. Hence, receivers subscribing to sublayers $L_{n, 0}$ to $L_{n, 2^{Q-1}}$ are forced to receive the probe packets at bit rate $R_{n, 2} Q-1$. By using delay trend detection, receivers can decide whether they have sufficient bandwidth to join the medium sublayer $L_{n, 2 Q-1}$, or stay at their original sublayer.

In the second step, the server sends the probe packets at the bit rates of first quarter and third quarter sublayers, $R_{n, 2^{Q}-2}$ (the bit rate of sublayer $\left.L_{n, 2} Q-2\right)$ and $R_{n, 3 \times 2^{Q-2}}$ (the bit rate of sublayer $L_{n, 3 \times 2^{Q-2}}$ ), simultaneously. During this probing stage, the packets of sublayers $L_{n, 0}$ to $L_{n, 2} Q-2$ are sent through sublayer $L_{n, 0}$ and the packets of sublayers $L_{n, 2^{Q-1}}$ to $L_{n, 3 \times 2^{Q-2}}$ are sent through sublayer $L_{n, 2 Q-1}$. This hierarchical sublayer probing is repeated until the server has probed the bit rates of all the odd sublayers $\left\{L_{n, j} \mid j=1,3, \ldots, M-1\right\}$.
Periodical probing of coarse layers and hierarchical probing of fine layers are used cooperatively in the scheme. Fig. 4 shows a sketch diagram of the hybrid coarse/fine layered probing scheme. The probing period of each layer depends on the probe size, packet size, and probing bit rate. Suppose K packets are sent during the probing period and the size of each packet is $\mathrm{S}$. For a given probing bit rate, $R_{t}$, the probing period required for a sender to transmit $\mathrm{K}$ packets is

$$
P_{t}=\frac{K \times S}{R_{t}} .
$$

For the $Q$ stage hierarchical sublayer probing in coarse layer $\mathrm{n}$, the probing period required in stage 1 is

$$
P_{n, 1}=\frac{K S}{R_{n, 2^{(Q-1)}}} .
$$

In stage 2 , the sender starts to probe the bit rates $R_{n, 2}{ }^{Q-2}$ and $R_{n, 3 \times 2^{Q-2}}$ simultaneously. The probing period in this stage is the maximum probing time required i.e.,

$$
P_{n, 2}=K S \times \max \left(\frac{1}{R_{n, 2^{(Q-2)}}}, \frac{1}{R_{n, 3 \times 2^{(Q-2)}}}\right)=\frac{K S}{R_{n, 2^{(Q-2)}}}
$$

since $R_{n, 3 \times 2^{(Q-2)}}>R_{n, 2^{(Q-2)}}$. Although $R_{n, 2^{Q-2}}$ and $R_{n, 3 \times 2^{Q-2}}$ are probed simultaneously, the probing periods are different. Suppose two receivers sharing the same link receive the probing bit rates $R_{n, 2^{Q-2}}$ and $R_{n, 3 \times 2^{Q-2}}$, respectively. If congestion occurs when bit rate $R_{n, 3 \times 2 Q-2}$ is received, it will affect the delay trend of the receiver receiving $R_{n, 2^{Q-2}}$; however, the latter can analyze the one-way delay trend before and after the period $K S / R_{n, 3 \times 2(Q-2)}$ to estimate whether it has the available bandwidth to join $R_{n, 2 Q-2}$.

The total time of one hierarchical probing cycle is defined as $P_{\text {tot }}^{H}$, and

$$
P_{\mathrm{tot}}^{H}=\sum_{s=1}^{Q}\left(\frac{K S}{R_{n, 2^{(Q-s)}}}+V\right)
$$

where $V$ denotes the waiting period between two probing stages.

\section{EXPERIMENTS}

In this section, we present the simulation results of the proposed hybrid coarse/fine layered multicast scheme using an ns-2 network simulator [11]. The results are compared with those of a layered multicast system based on the BIC control protocol [12]. A constant bit rate of UDP cross traffic is also introduced through the shared link to examine the effect of cross traffic. We also discuss the reconstructed video quality of the hybrid coarse/fine layered multicast framework.

\section{A. Network Simulations}

We implement the hybrid coarse/fine layered multicast scheme with six coarse layers, the bit rates of which are 64, $128,256,512,1024$, and $2048 \mathrm{kbps}$, respectively. Some of the layers are further divided into several fine-grain layers. Fig. 5 shows the bit rate allocation of each hybrid coarse/fine layer and the combined temporal, SNR, and spatial scalability corresponding to the hybrid coarse/fine layers. The simulation 


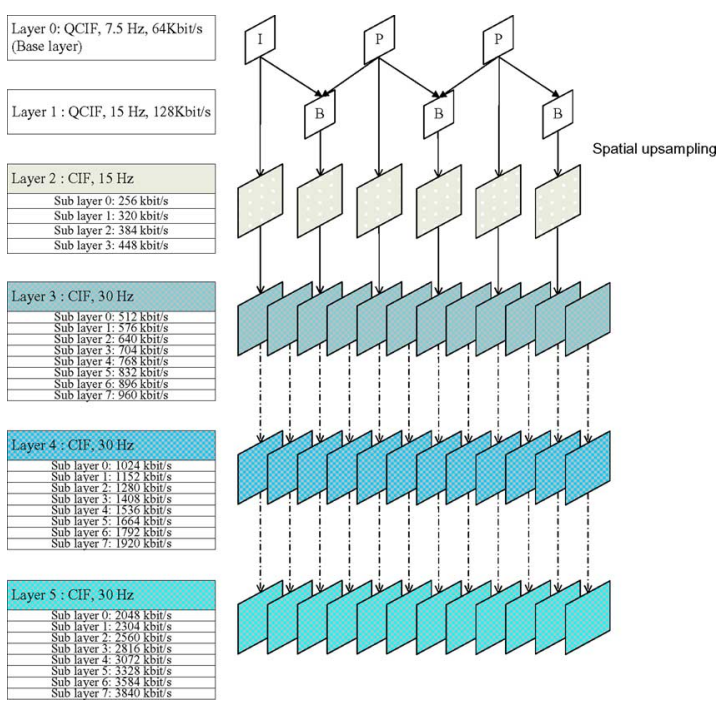

Fig. 5. Example of the bit rates of hybrid coarse/fine layers associated with their combined temporal, SNR, and spatial scalability.

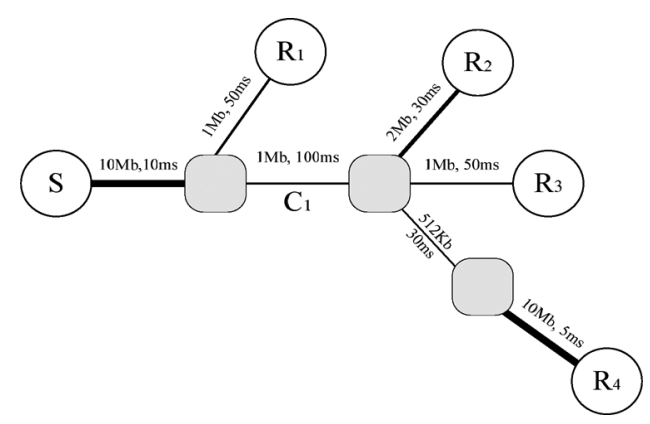

Fig. 6. Simulation topology.

network topology consists of one server and four receivers connected through different link capacities and round-trip times. All the links are connected by DropTail routers, as shown in Fig. 6 . The probe size is 50 packets, and the size of each packet is 512 bytes.

Fig. 7 shows the layer subscription of Receiver 3. The dashed line represents the layer subscription using the hierarchical probing scheme for hybrid coarse/fine layered multicast, and the solid line indicates the layer subscription using the BIC control protocol for traditional layered multicast. Since periodic coarse-layered probing takes a long time to finish a complete probing cycle, a start-up phase is applied to enable a new receiver to approach its optimal coarse layer subscription. The start-up phase is an aggressive process that allows a new receiver to join layers quickly before an increase in the delay trend is detected.

According to the simulation results, in the startup phase, Receiver 3 quickly subscribes to coarse layers up to coarse layer 4 and then leaves coarse layer 4 immediately due to packet loss. In traditional layered multicast based on the BIC congestion protocol, the sender periodically probes coarse layers. The bottleneck bandwidth of Receiver 3 is $1 \mathrm{Mbps}$; thus, Receiver 3 in traditional layered multicast stays steady at coarse layer 3 with a bit rate of $512 \mathrm{kbps}$. The dashed line in Fig. 7 shows the results of using our proposed hybrid coarse/fine layered multicast

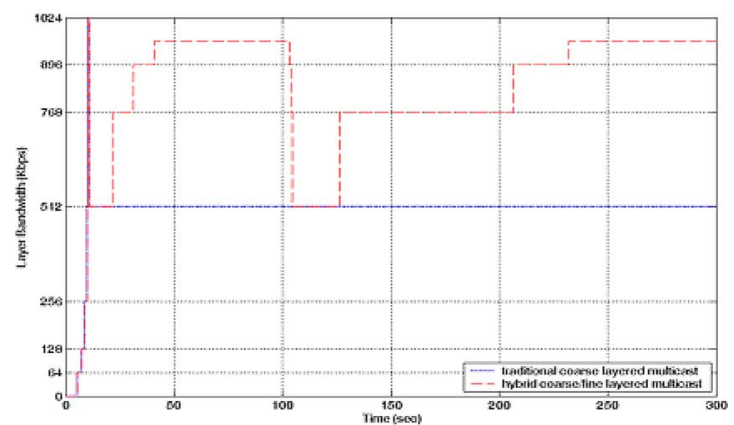

Fig. 7. Layer subscription of Receiver 3 with CBR cross traffic.

scheme. After periodically probing the coarse layers, the server uses the hierarchical sublayered probing scheme to probe the sublayers. Based on the probing of the sublayers, Receiver 3 joins sublayers 4, 6, and 7 of coarse layer 3 in sequence. After the hierarchical sublayered probing, Receiver 3 reaches its optimal sublayer with a bit rate of $960 \mathrm{kbps}$, which is very close to its maximum available bandwidth.

To determine the effect of cross traffic in the proposed scheme, we introduce a constant bit rate (CBR) of UDP cross traffic through the shared link $\mathbf{C}_{\mathbf{1}}$. The bit rate of the UDP cross traffic is $200 \mathrm{kbps}$ and the insertion period is between 100 and $200 \mathrm{~s}$. After $100 \mathrm{~s}$, the cross traffic starts to transmit the CBR packets through the shared link. Because the original traffic is transmitted at about $960 \mathrm{kbps}$, congestion occurs on the shared link. Due to the packet loss, Receiver 3 immediately drops the sublayers and stays at sublayer 0 of coarse layer 3 (512 kbps). Since the shared link's available bandwidth is now about $800 \mathrm{kbps}$, after the hierarchical probing, Receiver 3 joins sublayer 4 of coarse layer 3 again. When the cross traffic stops, Receiver 3 joins sublayers 6 and 7 of coarse layer 3 in turn after hierarchical sublayered probing and stays at sublayer 7 of coarse layer 3 with a bit rate of $960 \mathrm{kbps}$.

In contrast, in the traditional layered multicast, Receiver 3 based on the BIC control protocol stays at coarse layer 3 (sublayer 0) with a bit rate of $512 \mathrm{kbps}$. Because the maximum capacity on the shared link is about $1 \mathrm{Mbps}$, congestion does not occur when the cross traffic starts. Hence, Receiver 3 is not affected by the cross traffic and stays at sublayer 0 of coarse layer 3.

\section{B. Scalable Video Coding}

We now evaluate the coding efficiency of scalable video coding for different layers using a scalable video codec, SVC-Software_HHI_v02, to generate the scalable bit streams. The software, which was developed by the Fraunhofer Institute for Telecommunications, Heinrich Hertz Institute (HHI), is an implementation candidate for H.264/MPEG-4 SVC. The codec is a scalable extension of the H.264/AVC video coding standard based on the motion-compensated lifting scheme. Based on the bit rates of each layer applied in the network experiments, the combined temporal, SNR, and spatial scalability of the hybrid coarse/fine layered scheme is illustrated in Fig. 5.

Fig. 8 shows the reconstructed video quality of Receiver 3 using hybrid coarse/fine layered multicast (the solid curve marked by squares) compared to traditional coarse layered 


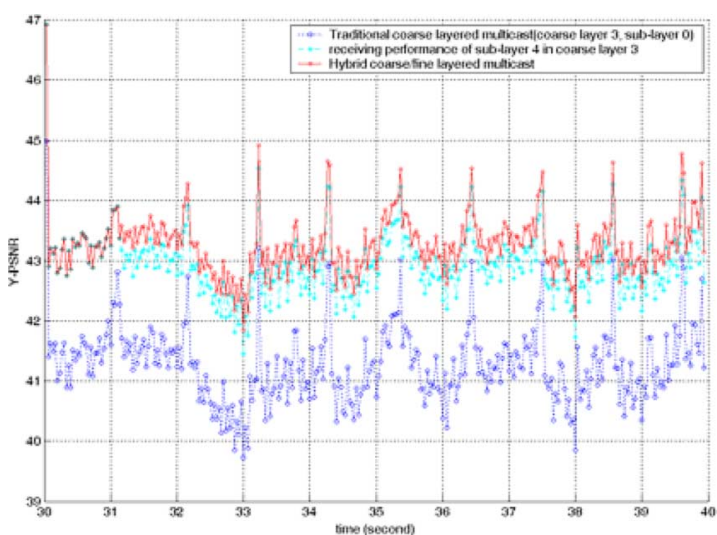

Fig. 8. Y-SPNR of a reconstructed video of Receiver 3 using the hybrid coarse/fine layered multicast compared to a traditional coarse layered multicast scheme. The encoded sequence is the News sequence.

multicast (the dotted curve marked by circles) between 30 and $40 \mathrm{~s}$. The dashed curve marked by stars represents the video quality achieved by sublayer 4 in coarse layer 3 . The receiver, using traditional layered multicast based on the BIC control scheme, subscribes at coarse layer 3 (sublayer 0), whereas the receiver using hybrid coarse/fine layered multicast based on the hierarchical probing scheme subscribes to sublayer 4 first and then subscribes to sublayer 6 in $31.1 \mathrm{~s}$. The Y-PSNR value of sublayer 4 is about $1.65 \mathrm{~dB}$ better than that of sublayer 0 , while the Y-PSNR value of sublayer 6 is about $2.03 \mathrm{~dB}$ better. According to the experiment results for scalable video coding, the reconstructed video quality of the receiver using hybrid coarse/fine layered multicast based on the hierarchical probing scheme is significantly better than that produced by traditional coarse-layered multicast.

Fig. 5 shows a simple example of the hybrid coarse/fine layered multicast framework. How to adjust the framework and the temporal, SNR, and spatial scalability to meet different scalability constraints for each fine-grain layer in order to achieve an optimal allocation is still an open issue. Because joining or leaving coarse layers affects the temporal, SNR, or spatial resolution, we should increase the threshold of the delay trend analysis to avoid frequent joining and leaving. However, changing the sublayer subscription will only affect the SNR quality, which human vision is less sensitive to. Therefore, we can reduce the threshold of the delay trend in sublayer probing so that receivers can locate their optimal sublayer quickly, depending on variations in the available bandwidth.

\section{CONCLUSION}

We have presented a new framework that uses delay trend detection with a hierarchical layered probing scheme for band- width inference congestion control of hybrid coarse/fine layered multicast. Through hierarchical probing of the coarse layers and their sublayers, a receiver can rapidly adapt to the optimal finegrain layer based on the rapidly changing bandwidth. We propose an effective hybrid coarse/fine layered multicast scheme that is based on a combination of the hierarchical bandwidth inference congestion control scheme and scalable video coding based on MCTF. The experiment results of network simulation and coding efficiency show that the proposed hybrid coarse/fine layered multicast scheme can achieve efficient scalable multimedia streaming over heterogeneous networks.

\section{REFERENCES}

[1] H. Schwarz, D. Marpe, and T. Wiegand, Scalable Extension of H.264/AVC, ISO/IEC JTC1/SC29/WG112 Doc.M10569, Mar. 2004.

[2] Scalable Extension of H.264/AVC, ISO/IEC JTC1/WG11 MPEG04/ M10569/S03, ISO/IEC, Mar. 2004.

[3] , Joint Video Team (JVT) of ISO/IEC MPEG \& ITU-T VCEG JVTO202, "Joint Scalable Video Model JSVM 2," in 15th Meeting, Busan, KR, Apr. 16-22, 2005.

[4] J. Byers, M. Frumin, G. Horn, M. Luby, M. Mitzenmacher, A. Roetter, and W. Shaver, "FLID-DL: Congestion control for layered multicast," in Proc. 2nd Int. Workshop Group Communication (NGC 2000), Stanford, CA, Nov. 2000, pp. 71-81.

[5] J.-L. Lin, W.-L. Hwang, and S.-C. Pei, "SNR scalability based on bitplane coding of matching pursuit atoms at low bit rates: Fine-grained and two-layer," IEEE Trans. Circuits Syst. Video Technol., vol. 15, no. 1, pp. 3-14, Jan. 2005.

[6] J.-R. Ohm, M. van der Schaar, and J. W. Woods, "Interframe wavelet coding-motion picture representation for universal scalability," Signal Process.: Image Commun., vol. 19, no. 9, pp. 877-908, Oct. 2004.

[7] J. Xu, S. Li, and Y.-Q. Zhang, "A wavelet codec using 3-D ESCOT," presented at the PCM2000, Dec. 2000.

[8] K. Nakauchi, H. Morikawa, and T. Aoyama, "Network-supported rate control mechanism for multicast streaming media," in Proc. Sym. Appl. Internet 2001, Jan. 2001, pp. 131-138.

[9] L. Vicisano, L. Rizzo, and J. Crowcroft, "TCP-like congestion control for layered multicast data transfer," presented at the INFOCOM'98, San Francisco, CA, Mar. 1998.

[10] L. Wu, R. Sharma, and B. Smith, "Thin streams: An architecture for multicasting layered video," in Proc. NOSSDAV'97, May 1997, pp. 173-182.

[11] NS-2, The Network Simulator, 2008 [Online]. Available: http://www. isi.edu/nsnam $/ \mathrm{ns}$

[12] Q. Liu, "Available bandwidth estimation and rate-based congestion control in multimedia communication," Ph.D. dissertation, Univ. Washington, Seattle, May 2004.

[13] Q. Liu, J.-J. Yoo, B.-T. Jang, K.-H. Choi, and J.-N. Hwang, "A scalable VideoGIS system for GPS-guided vehicles," Signal Process.: Image Commun., vol. 20, no. 3, pp. 205-208, Mar. 2005.

[14] S. McCanne, V. Jacobson, and M. Vetterli, "Receiver-driven layered multicast," in Proc. ACM SIGCOMM, Aug. 1996, pp. 117-130.

[15] W. Li, "Overview of fine granularity scalability in MPEG-4 video standard," IEEE Trans. Circuits Syst. Video Technol., vol. 11, pp. 301-317, 2001.

[16] X. Li, S. Paul, and M. H. Ammar, "Multi-session rate control for layered video multicast," presented at the Multimedia Computing Netw., Jan. 1999. 